\title{
ON THE DERIVATION OF THE SCHRÖDINGER EQUATION WITH POINT-LIKE NONLINEARITY
}

\author{
C. Cacciapuoti \\ Dipartimento di Scienza e Alta Tecnologia, Università dell'Insubria, \\ Via Valleggio 11, 22100 Como, Italy \\ claudio.cacciapuoti@uninsubria.it
}

PACS 02.30.Jr, 03.65.Db, 02.30.Rz DOI 10.17586/2220-8054-2015-6-1-79-94

In this report we discuss the problem of approximating nonlinear delta-interactions in dimensions one and three with regular, local or non-local nonlinearities. Concerning the one dimensional case, we discuss a recent result proved in [10], on the derivation of nonlinear delta-interactions as limit of scaled, local nonlinearities. For the three dimensional case, we consider an equation with scaled, non-local nonlinearity. We conjecture that such an equation approximates the nonlinear delta-interaction, and give an heuristic argument to support our conjecture.

Keywords: Nonlinear Schrödinger equation, nonlinear delta interactions, zero-range limit of concentrated nonlinearities.

Received: 15 January 2015

\section{Introduction}

Point interactions in quantum mechanics describe the dynamics of particles in interaction with potentials supported on a (finite or infinite) set of points. In dimension one, they are quite easily understood as a limit of short range potentials and they have been a subject of study since the very early years of quantum mechanics, a most well-known application is the Kronig-Penney model [17], which describes the dynamics of electrons in crystals. In higher dimensions, the first attempts to use point interaction came from nuclear physics, to describe the short range interactions between nucleons. It was found that to define a potential supported in one point in dimension larger than one is a subtler and less intuitive matter. Indeed, a rigorous mathematical definition of point potentials in dimension three was achieved only in the 1960's with the work of Berezin and Faddeev [7].

At a formal level the Hamiltonian describing a particle in a potential supported by points can be written as: $H_{\alpha}=-\Delta+\sum_{j} \alpha_{j} \delta_{y_{j}}$, where $\alpha=\left\{\alpha_{j}\right\}$ is a set of assigned real constants, $Y=\left\{y_{j}\right\}$ is a fixed set of points in $\mathbb{R}^{d}$, and $\delta_{y_{j}}$ is the Dirac delta-distribution centered in $y_{j}$. For this reason, $H_{\alpha}$ is also referred to as delta-interaction. For a thorough discussion on the Hamiltonian $H_{\alpha}$ see [6], the monograph also includes an historical overview of the topic and an appendix with an update on the progresses in the area.

The subject of this report is the Schrödinger equation associated with a nonlinear version of the Hamiltonian $H_{\alpha}$ in which the constants $\alpha_{j}$ depend on the state of the system. Such an equation describes the propagation of a wave function in a medium whose response is nonlinear only in some isolated points. The question that we want to address is whether the solution of the equation with point-like nonlinearities is approximated by the solution of an equation with smooth nonlinear terms.

We shall focus attention only on the one and three dimensional cases. They will be discussed separately, with the related applications and literature, in Sec. 2 and 3 respectively. 
In dimension one, a solution to the approximating problem was given in [10]. Here we shall briefly discuss the result and give a sketch of the proof, we refer to [10] for the details.

In dimension three, the problem is still open. Here we shall exhibit an equation that we conjecture to approximate the nonlinear delta-interaction, and give an heuristic argument to support our conjecture.

To the best of our knowledge very little is known about the two dimensional case. Also the correct definition of the limit problem, and the study of the well-posedness, is not yet done. In dimension four or higher, instead, the problem cannot be set up because is not possible to define the Hamiltonian $H_{\alpha}$, see [6].

In what follows we shall construct the approximating equation by introducing a small scaling parameter that will be denoted by $\varepsilon$. We shall denote by $c$ a generic positive constant whose value may change from line to line. The constant $c$ may depend on the parameters entering the equations and on the initial data, but in no case $c$ will depend on the scaling parameter.

\section{Nonlinear delta-interactions in dimension one}

In this section we shall present a review on the Schrödinger equation with delta-like nonlinearities concentrated in a fixed set of points in dimension one. We shall recall the definition of the equation and the basic results about its well-posedness, this part is a survey of the works $[4,5]$ and [16]. Then we shall state the main result of [10] on the derivation of the equation as the limit of Schrödinger equations with spatially non-homogeneous scaled nonlinearity, and discuss the basic ideas behind the proof. We shall also mention a different approximation method based on non-local smoothed nonlinearities, this method was used in [16] as an intermediate step in the proof of the well-posedness of the limit equation.

To begin, it is worth recalling the definition of the operator $H_{\alpha}$ in dimension one.

The domain of the self-adjoint operator $H_{\alpha}$ is

$$
D\left(H_{\alpha}\right)=\left\{\psi \in H^{2}(\mathbb{R} \backslash Y) \cap H^{1}(\mathbb{R}): \psi^{\prime}\left(y_{j}^{+}\right)-\psi^{\prime}\left(y_{j}^{-}\right)=\alpha_{j} \psi\left(y_{j}\right)\right\} .
$$

The operator $H_{\alpha}$ acts on the elements in its domain as the Laplacian, everywhere, except that in the points $y_{j}$, i.e.,

$$
H_{\alpha} \psi=-\psi^{\prime \prime} \quad \forall x \neq y_{j}, j=1, \ldots, N
$$

for any $\psi \in D\left(H_{\alpha}\right)$.

The form domain of $H_{\alpha}$ does not depend on the parameters $\alpha_{j}$ and coincides with $H^{1}(\mathbb{R})$.

To give a precise definition of the nonlinear dynamics in which we are interested, we start with a discussion on the non-autonomous Hamiltonian $H_{\alpha(t)}$, for which we let the parameters $\alpha_{j}$ depend on the time variable $t$. Such Hamiltonians are interesting on their own both from the physical and mathematical point of view, see, e.g., [15,19] and references therein.

When $\alpha_{j}(t)$ are assigned real valued functions of time, the domain $D\left(H_{\alpha(t)}\right)$ changes in time as well. In particular, if $\psi(t) \in D\left(H_{\alpha(t)}\right)$, then $\psi(t)$ must satisfy the time dependent jump conditions $\psi^{\prime}\left(t, y_{j}^{+}\right)-\psi^{\prime}\left(t, y_{j}^{-}\right)=\alpha_{j}(t) \psi\left(t, y_{j}\right)$. Conversely, the form domain of $H_{\alpha(t)}$ does not depend on $t$ and coincides with $H^{1}(\mathbb{R})$. 
Consider the Cauchy problem

$$
\left\{\begin{array}{l}
i \frac{d}{d t} \psi(t)=H_{\alpha(t)} \psi(t) \\
\left.\psi\right|_{t=0}=\psi_{0}
\end{array}\right.
$$

The weak solutions of (3) are the solutions of the associated integral equation

$$
\psi(t, x)=\left(U(t) * \psi_{0}\right)(x)-i \sum_{j=1}^{N} \int_{0}^{t} d s U\left(t-s, x-y_{j}\right) \alpha_{j}(s) \psi\left(s, y_{j}\right),
$$

where $U(t)$ is the unitary evolution group associated to the free Laplacian in dimension one, its explicit expression is given by

$$
U(t, x)=\frac{e^{i \frac{x^{2}}{4 t}}}{(4 \pi i t)^{1 / 2}} .
$$

The solutions of (4) belong to the domain of $H_{\alpha(t)}$ only under suitable assumptions on the initial datum and on the regularity of the functions $\alpha_{j}(t)$. For a discussion on this problem we refer to the works $[15,19]$.

From (4), it is clear that the solution $\psi(t, x)$ is fully determined from the quantities $\psi\left(t, y_{j}\right), j=1, \ldots, N$. Then, by evaluating the equation (4) in $y_{k}, k=1, \ldots, N$, the problem of finding $\psi(t, x)$ can be reduced to solve a system of $N$ coupled Volterra equations for the functions $\psi\left(t, y_{k}\right)$. This fact will also be used in the analysis of the nonlinear problem.

To define the nonlinear delta-interactions we use the weak formulation (4) and mimic the nonlinear flow on it by letting $\alpha_{j}(t) \rightarrow \alpha_{j}\left(\left|\psi\left(t, y_{j}\right)\right|^{2}\right)$. In this way we give a weak formulation of the problem given by the equation $i \partial_{t} \psi=-\partial_{x x}^{2} \psi+\sum_{j=1}^{N} \alpha_{j}\left(\left|\psi\left(t, y_{j}\right)\right|^{2}\right) \delta_{y_{j}} \psi$ with initial datum $\psi_{0}$. We remark that the choice of the nonlinearity $\alpha_{j}\left(\left|\psi\left(t, y_{j}\right)\right|^{2}\right)$ guarantees that the equation is invariant under phase multiplication and that the nonlinearity is local; strong solutions should satisfy the jump condition $\psi^{\prime}\left(t, y_{j}^{+}\right)-\psi^{\prime}\left(t, y_{j}^{-}\right)=\alpha_{j}\left(\left|\psi\left(t, y_{j}\right)\right|^{2}\right) \psi\left(t, y_{j}\right)$.

We shall follow $[4,5]$ and consider only power-type nonlinearities, i.e., $\alpha_{j}(z)=\gamma_{j} z^{\mu_{j}}$ for some real constants $\gamma_{j}$, and $\mu_{j}>0$, but more general nonlinearities could be considered, see, e.g., [16]. Our model nonlinear delta-interaction is then defined by the integral equation

$$
\psi(t, x)=\left(U(t) * \psi_{0}\right)(x)-i \sum_{j=1}^{N} \gamma_{j} \int_{0}^{t} d s U\left(t-s, x-y_{j}\right)\left|\psi\left(s, y_{j}\right)\right|^{2 \mu_{j}} \psi\left(s, y_{j}\right) .
$$

This is a weak formulation of the problem

$$
\left\{\begin{array}{l}
i \frac{\partial}{\partial t} \psi(t, x)=-\frac{\partial^{2}}{\partial x^{2}} \psi(t, x)+\sum_{j=1}^{N} \gamma_{j} \delta_{y_{j}}\left|\psi\left(t, y_{j}\right)\right|^{2 \mu_{j}} \psi(t, x) \\
\psi(0, x)=\psi_{0}(x)
\end{array}\right.
$$

Nonlinear point interactions of this form have been used in solid state physics, see, e.g., $[8,18]$ (and references therein), and, more recently, to model nonlinear periodic systems, such as Bose-Einstein condensates trapped into optical lattices, see [14].

As for the time dependent delta-interactions, we do not address here the problem of showing under what conditions on $\gamma_{j}, \mu_{j}$ and $\psi_{0}$, the solution of (5) satisfies the jump condition $\psi^{\prime}\left(t, y_{j}^{+}\right)-\psi^{\prime}\left(t, y_{j}^{-}\right)=\gamma_{j}\left|\psi\left(t, y_{j}\right)\right|^{2 \mu_{j}} \psi\left(t, y_{j}\right)$. Instead we take initial data in the form domain, $H^{1}(\mathbb{R})$, and discuss the well-posedness and the approximation problem in $H^{1}(\mathbb{R})$. 
We start by recalling that global well-posedness of (5) for initial data in $H^{1}(\mathbb{R})$ and under the condition that

$$
\mu_{j}<1 \quad \text { if } \quad \gamma_{j}<0
$$

was proved in [5] (see also [16] for different kind of nonlinearities).

The proof of global well-posedness as given in [5] follows a standard scheme: first prove local well-posedness; then show that there exist some conserved quantities; finally, extend the well-posedness to an arbitrarily large time $T$ by exploiting the conserved quantities together with results on the existence of global solutions for the Volterra equations. We shall not comment on the first step of the proof, the analysis of the local well-posedness. For the purposes of this report it is enough to discuss the conservation laws and how they affect the proof of the global well-posedness, the discussion will make clear where the condition (6) plays a role.

For any initial state in $H^{1}(\mathbb{R})$, there exist two quantities which are conserved by the nonlinear flow (5): the $L^{2}$-norm (also referred to as mass), and the energy

$$
E[\psi]=\left\|\psi^{\prime}\right\|^{2}+\sum_{j=1}^{N} \frac{\gamma_{j}}{\mu_{j}+1}\left|\psi\left(y_{j}\right)\right|^{2 \mu_{j}+2} .
$$

Then, if $\psi(t)$ is the solution of (5) with $\psi_{0} \in H^{1}(\mathbb{R})$, one has that $\|\psi(t)\|^{2}=\left\|\psi_{0}\right\|^{2}$ and $E[\psi(t)]=E\left[\psi_{0}\right]$.

One main issue in the proof of the global well-posedness is to prove that the $H^{1}$-norm of the solution does not blow-up in finite time, i.e., that there does not exist $T$ such that $\lim \sup _{t \rightarrow T}\|\psi(t)\|_{H^{1}}<\infty$.

From the conservation of mass, proving that blow-up does not occur is reduced to prove that the kinetic energy $\left\|\psi^{\prime}(t)\right\|^{2}$ does not blow-up in finite time.

If $\gamma_{j} \geqslant 0$ for all $j$, the conservation of the energy immediately implies that $\left\|\psi^{\prime}(t)\right\| \leqslant c$ (as well as $\left|\psi\left(t, y_{j}\right)\right| \leqslant c$ ). Then no restriction on the power of the nonlinearity is needed.

If for some $j, \gamma_{j}<0$, then the conservation of the energy alone is not enough to guarantee that $\left\|\psi^{\prime}(t)\right\|$ stays bounded. One needs to be sure that in the energy the growth of $\left\|\psi^{\prime}(t)\right\|$ cannot be compensated by the negative term $\sum_{j: \gamma_{j}<0} \frac{\gamma_{j}}{\mu_{j}+1}\left|\psi\left(t, y_{j}\right)\right|^{2}$. This is achieved by using the well known Gagliardo-Nirenberg inequality

$$
\|\psi\|_{\infty} \leqslant c\left\|\psi^{\prime}\right\|^{\frac{1}{2}}\|\psi\|^{\frac{1}{2}}
$$

Inequality (7), together with the energy and mass conservation, imply

$$
\begin{aligned}
E\left[\psi_{0}\right]=E[\psi(t)] & =\left\|\psi^{\prime}(t)\right\|^{2}+\sum_{j: \gamma_{j} \geqslant 0} \frac{\gamma_{j}}{\mu_{j}+1}\left|\psi\left(t, y_{j}\right)\right|^{2 \mu_{j}+2}-\sum_{j: \gamma_{j}<0} \frac{\left|\gamma_{j}\right|}{\mu_{j}+1}\left|\psi\left(t, y_{j}\right)\right|^{2 \mu_{j}+2} \\
& \geqslant\left\|\psi^{\prime}(t)\right\|^{2}-c \sum_{j: \gamma_{j}<0}\left\|\psi^{\prime}(t)\right\|^{\mu_{j}+1} .
\end{aligned}
$$

The bound (8) makes clear where the condition (6) comes from: if $\mu_{j}<1$ for all the $j$ 's such that $\gamma_{j}<0$, then the inequality (8) implies that $\left\|\psi^{\prime}(t)\right\| \leqslant c$; otherwise the negative term at the r.h.s. dominates for $\left\|\psi^{\prime}(t)\right\|$ large, and the inequality (8) does not imply that $\left\|\psi^{\prime}(t)\right\|$ is bounded.

We note that in [5], it is shown that for $N=1$ the condition (6) is indeed sharp. In the sense that for $\mu \equiv \mu_{1} \geqslant 1$, it is possible to find an initial datum $\psi_{0} \in H^{1}(\mathbb{R})$ such that there exists $t_{c}<\infty$ for which $\lim \sup _{t \rightarrow t_{c}}\|\psi(t)\|_{H^{1}(\mathbb{R})}=\infty$, i.e., the solution blows-up in finite time. 


\subsection{Point-like limit of scaled spatially non-homogeneous nonlinearities in dimension one}

Here we discuss the approximating equation used in [10] and give a sketch of the proof of the convergence of the solutions.

For any $\varepsilon>0$, consider the nonlinear flow

$$
\psi^{\varepsilon}(t, x)=\left(U(t) * \psi_{0}\right)(x)-i \sum_{j=1}^{N} \int_{0}^{t} d s\left(U(t-s) * V_{j}^{\varepsilon}\left(\cdot-y_{j}\right)\left|\psi^{\varepsilon}(s)\right|^{2 \mu_{j}} \psi^{\varepsilon}(s)\right)(x)
$$

where

$$
V_{j}^{\varepsilon}(x)=\frac{1}{\varepsilon} V_{j}\left(\frac{x}{\varepsilon}\right) \quad \text { with } \quad V_{j} \in L^{1}(\mathbb{R},(1+|x|) d x) \cap L^{\infty}(\mathbb{R})
$$

and $\mu_{j}>0$.

We remark that Eq. (9) is a weak formulation of the Cauchy problem

$$
\left\{\begin{array}{l}
i \frac{\partial}{\partial t} \psi^{\varepsilon}(t, x)=-\frac{\partial^{2}}{\partial x^{2}} \psi^{\varepsilon}(t, x)+\sum_{j=1}^{N} V_{j}^{\varepsilon}\left(x-y_{j}\right)\left|\psi^{\varepsilon}(t, x)\right|^{2 \mu_{j}} \psi^{\varepsilon}(t, x) \\
\psi^{\varepsilon}(0, x)=\psi_{0}(x)
\end{array}\right.
$$

This describes a situation in which there is a spacial inhomogeneity of the response of the medium to the wave function propagation. The regions in which the response is nonlinear are supported on intervals of length of order $\varepsilon$ around the points $y_{j}$.

Before discussing the convergence of the solutions of Eq. (9) to the solutions of Eq. (5) we shall comment on the well-posedness of (9). Under the assumption (10), Corollary 6.1.2 of [11] applies, so that, for any $\varepsilon>0$, one has global existence of strong $H^{1}$-solutions for every initial datum $\psi_{0} \in H^{1}(\mathbb{R})$ for any $\mu_{j}>0$ if $V_{j} \geqslant 0$ (defocusing case) and for $0<\mu_{j}<2$ if $V_{j}$ is negative in some open interval (for $\mu_{j}=2$, the critical case, one has global existence for small data, see Remark 6.1.3 of [11]).

As for the limit problem (5), to prove global well-posedness in $H^{1}$ one uses the fact that there exist two conserved quantities: the $L^{2}$-norm (mass) and the energy

$$
E^{\varepsilon}[\psi]=\left\|\psi^{\prime}\right\|^{2}+\sum_{j=1}^{N} \frac{1}{\mu_{j}+1} \int_{\mathbb{R}} V_{j}^{\varepsilon}\left(y-y_{j}\right)|\psi(y)|^{2 \mu+2} d y .
$$

Precisely one has that, for any $\psi_{0} \in H^{1}(\mathbb{R})$, the solution $\psi^{\varepsilon}(t)$ of $(9)$ is such that:

$$
\left\|\psi^{\varepsilon}(t)\right\|^{2}=\left\|\psi_{0}\right\|^{2} \quad \text { and } \quad E\left[\psi^{\varepsilon}(t)\right]=E\left[\psi_{0}\right] .
$$

From the mass conservation, to prove that the $H^{1}$-norm of $\psi^{\varepsilon}(t)$ does not blow-up in finite time, one needs to show that the quantity $\left\|\psi^{\varepsilon^{\prime}}(t)\right\|$ stays bounded for any $t$. This is achieved by using the following argument, write $V_{j}$ as the sum of its positive and negative part, $V_{j}=V_{j,+}-V_{j,-}$, with $V_{j,+} \geqslant 0$ and $V_{j,-}>0$. Then, by the Gagliardo-Nirenberg inequality $(7)$

$$
\begin{aligned}
\frac{1}{\mu_{j}+1} \int_{\mathbb{R}} V_{j}^{\varepsilon}\left(y-y_{j}\right)\left|\psi^{\varepsilon}(y)\right|^{2 \mu_{j}+2} d y & =\frac{1}{\mu_{j}+1} \int_{\mathbb{R}} \frac{1}{\varepsilon} V_{j}\left(\frac{y-y_{j}}{\varepsilon}\right)\left|\psi^{\varepsilon}(y)\right|^{2 \mu_{j}+2} d y \\
& \geqslant-\frac{1}{\mu_{j}+1} \int_{\mathbb{R}} \frac{1}{\varepsilon} V_{j,-}\left(\frac{y-y_{j}}{\varepsilon}\right)\left|\psi^{\varepsilon}(y)\right|^{2 \mu_{j}+2} d y \\
& \geqslant-\frac{c}{\varepsilon}\left\|\psi^{\varepsilon}\right\|_{\infty}^{2 \mu_{j}} \int_{\mathbb{R}}\left|\psi^{\varepsilon}(y)\right|^{2} d y \geqslant-\frac{c}{\varepsilon}\left\|\psi^{\varepsilon \prime}\right\|^{\mu_{j}}\left\|\psi^{\varepsilon}\right\|^{2}
\end{aligned}
$$


We denote by $\mathcal{K} \subseteq\{1, \ldots, N\}$ the set of indices $j$ such that $V_{j}$ is negative in some open interval. Then by mass and energy conservation, and the inequality above, one has that,

$$
E^{\varepsilon}\left[\psi_{0}\right]=E^{\varepsilon}\left[\psi^{\varepsilon}(t)\right] \geqslant\left\|\psi^{\varepsilon^{\prime}}(t)\right\|^{2}-\frac{c}{\varepsilon} \sum_{j \in \mathcal{K}}\left\|\psi^{\varepsilon^{\prime}}(t)\right\|^{\mu_{j}} .
$$

As a consequence, one has $\left\|\psi^{\varepsilon^{\prime}}(t)\right\| \leqslant C_{\varepsilon}$ if $\mu_{j}<2$ for all $j \in \mathcal{K}$. We remark that the argument used above gives a bound on $\left\|\psi^{\varepsilon^{\prime}}(t)\right\|$ which is not uniform in $\varepsilon$. The constant $C_{\varepsilon}$ depends on $\varepsilon$ because $\varepsilon$ appears at the denominator of $c$ in Eq. (12). This is enough to obtain global well-posedness for any $\varepsilon>0$ but it is not enough to prove the convergence result. For that we shall need a bound on $\left\|\psi^{\varepsilon^{\prime}}(t)\right\|$ which is uniform in $\varepsilon$, we shall be able to obtain it at the cost of a more restrictive constraint on $\mu_{j}$.

Now we are ready to state the main result of [10].

Theorem 2.1. For all $j=1, \ldots, N$, take $V_{j}^{\varepsilon}$ as in (10) and $0<\mu_{j}<1$ if $V_{j}$ is negative in some open interval. For any $\psi_{0} \in H^{1}(\mathbb{R})$, let $\psi$ be the solution of Eq. (5) with $\gamma_{j}=\int_{\mathbb{R}} V_{j} d x$ and $\psi^{\varepsilon}$ be the solution of Eq. (9). Then, for any $T>0$,

$$
\lim _{\varepsilon \rightarrow 0} \sup _{t \in[0, T]}\left\|\psi(t)-\psi^{\varepsilon}(t)\right\|_{H^{1}(\mathbb{R})}=0 .
$$

Outline of the proof of Th. 2.1. We shall discuss only the main ideas behind the proof of Th. (2.1), for the details we refer to [10]. We divide the proof in four steps.

Step 1. The first step is to obtain a bound for $\left\|\psi^{\varepsilon^{\prime}}(t)\right\|$ which is uniform in $\varepsilon$. This can be achieved at the cost of a more restrictive constraint on the power of the nonlinear term, precisely we shall need to assume that $\mu_{j}<1$, for all $j \in \mathcal{K}$. Writing, as in the proof of the well-posedness of Eq. (9), $V_{j}$ as the sum of its positive and negative parts, and by assumption (10) and Gagliardo-Nirenberg inequality, we have that

$$
\begin{aligned}
\frac{1}{\mu_{j}+1} \int_{\mathbb{R}} V_{j}^{\varepsilon}\left(y-y_{j}\right)\left|\psi^{\varepsilon}(y)\right|^{2 \mu_{j}+2} d y & =\frac{1}{\mu_{j}+1} \int_{\mathbb{R}} \frac{1}{\varepsilon} V_{j}\left(\frac{y-y_{j}}{\varepsilon}\right)\left|\psi^{\varepsilon}(y)\right|^{2 \mu_{j}+2} d y \\
& \geqslant-\frac{1}{\mu_{j}+1} \int_{\mathbb{R}} V_{j,-}(y)\left|\psi^{\varepsilon}\left(y_{j}+\varepsilon y\right)\right|^{2 \mu_{j}+2} d y \\
& \geqslant-c\left\|\psi^{\varepsilon \prime}\right\|^{\mu_{j}+1}
\end{aligned}
$$

The latter bound together with the conservation of the energy, gives

$$
E^{\varepsilon}\left[\psi_{0}\right]=E^{\varepsilon}\left[\psi^{\varepsilon}(t)\right] \geqslant\left\|\psi^{\varepsilon^{\prime}}(t)\right\|^{2}-c \sum_{j \in \mathcal{K}}\left\|\psi^{\varepsilon^{\prime}}(t)\right\|^{\mu_{j}+1} .
$$

Since

$$
\sup _{\varepsilon \in[0,1]} E^{\varepsilon}\left[\psi_{0}\right] \leqslant\left\|\psi_{0}^{\prime}\right\|^{2}+\left\|\psi_{0}\right\|_{\infty}^{2 \mu+2} \sum_{j=1}^{N} \frac{1}{\mu_{j}+1} \int_{\mathbb{R}} d x V_{j,+}(x) \equiv K
$$

we finally get the inequality:

$$
\left\|\psi^{\varepsilon \prime}(t)\right\|^{2}-c \sum_{j \in \mathcal{K}}\left\|\psi^{\varepsilon \prime}(t)\right\|^{\mu_{j}+1} \leqslant K
$$

which implies $\left\|\psi^{\varepsilon^{\prime}}(t)\right\| \leqslant c$ if $\mu_{j}<1$ for all $j \in \mathcal{K}$ and $t>0$, by the Gagliardo-Nirenberg inequality, this also implies the bound $\left\|\psi^{\varepsilon}(t)\right\|_{\infty} \leqslant c$.

Step 2. The solution of the limit problem (5) is completely determined by the values $\psi\left(t, y_{j}\right), j=1, \ldots, N$. Then to find $\psi(t, x)$ we must solve first the system of coupled nonlinear 
On the derivation of the Schrödinger equation with point-like nonlinearity

Volterra equations in the variables $\psi\left(t, y_{k}\right)$.

$$
\psi\left(t, y_{k}\right)=\left(U(t) * \psi_{0}\right)\left(y_{k}\right)-i \sum_{j=1}^{N} \gamma_{j} \int_{0}^{t} d s U\left(t-s, y_{k}-y_{j}\right)\left|\psi\left(s, y_{j}\right)\right|^{2 \mu_{j}} \psi\left(s, y_{j}\right),
$$

$k=1, \ldots, N$.

For this reason in the second step of the proof we address the problem of showing the convergence of $\psi^{\varepsilon}\left(t, y_{k}\right)$ to $\psi\left(t, y_{k}\right)$, for all $k=1, \ldots, N$. To this end, we compute $\psi^{\varepsilon}\left(t, y_{k}\right)$ by using Eq. (9), we get

$$
\psi^{\varepsilon}\left(t, y_{k}\right)=\left(U(t) * \psi_{0}\right)\left(y_{k}\right)-i \sum_{j=1}^{N} \int_{0}^{t} d s\left(U(t-s) * V_{j}^{\varepsilon}\left(\cdot-y_{j}\right)\left|\psi^{\varepsilon}(s)\right|^{2 \mu_{j}} \psi^{\varepsilon}(s)\right)\left(y_{k}\right) .
$$

We rewrite the latter equation in a more explicit form as

$$
\begin{aligned}
& \psi^{\varepsilon}\left(t, y_{k}\right)=\left(U(t) * \psi_{0}\right)\left(y_{k}\right) \\
& \quad-i \sum_{j=1}^{N} \int_{0}^{t} d s \int_{\mathbb{R}} U\left(t-s, y_{k}-y_{j}-\varepsilon y\right) V_{j}(y)\left|\psi^{\varepsilon}\left(s, y_{j}+\varepsilon y\right)\right|^{2 \mu_{j}} \psi^{\varepsilon}\left(s, y_{j}+\varepsilon y\right) d y .
\end{aligned}
$$

Letting $\varepsilon \rightarrow 0$ at the r.h.s. of the latter equation one would expect that

$$
\psi^{\varepsilon}\left(t, y_{k}\right) \simeq\left(U(t) * \psi_{0}\right)\left(y_{k}\right)-i \sum_{j=1}^{N} \int_{\mathbb{R}} V_{j}(y) d y \int_{0}^{t} d s U\left(t-s, y_{k}-y_{j}\right)\left|\psi^{\varepsilon}\left(s, y_{j}\right)\right|^{2 \mu_{j}} \psi^{\varepsilon}\left(s, y_{j}\right) .
$$

By comparison with Eq. (13) one notices that $\psi^{\varepsilon}\left(t, y_{k}\right)$ approximatively satisfies the same equation as $\psi\left(t, y_{k}\right)$ if $\gamma_{j}=\int_{\mathbb{R}} V_{j}(y) d y$. To make precise this idea we subtract Eq. (13) from (14). By adding and subtracting suitable terms at the r.h.s. (using the fact that $\left.\gamma_{j}=\int_{\mathbb{R}} V_{j}(y) d y\right)$, we end up with the identity

$$
\begin{aligned}
& \psi^{\varepsilon}\left(t, y_{k}\right)-\psi\left(t, y_{k}\right)= \\
& \quad-i \sum_{j=1}^{N} \gamma_{j} \int_{0}^{t} d s U\left(t-s, y_{k}-y_{j}\right)\left(\left|\psi^{\varepsilon}\left(s, y_{j}\right)\right|^{2 \mu_{j}} \psi^{\varepsilon}\left(s, y_{j}\right)-\left|\psi\left(s, y_{j}\right)\right|^{2 \mu_{j}} \psi\left(s, y_{j}\right)\right)+\mathcal{R}^{\varepsilon}(t)
\end{aligned}
$$

where $\mathcal{R}^{\varepsilon}(t)$ is a remainder that satisfies the bound $\sup _{t \in[0, T]}\left|\mathcal{R}^{\varepsilon}(t)\right| \leqslant c \varepsilon^{\delta}$ for any $0<\delta<1 / 2$ and $T>0$, we refer to [10] for the details. Here we use the a priori bounds $\|\psi(t)\|_{\infty} \leqslant c$ and $\left\|\psi^{\varepsilon}(t)\right\|_{\infty} \leqslant c$.

From the bound for the remainder $\mathcal{R}^{\varepsilon}(t)$, the explicit expression of $U(t, x)$, the fact that $\left.|| a\right|^{2 \mu} a-|b|^{2 \mu} b\left|\leqslant\left(|a|^{2 \mu}+|b|^{2 \mu}\right)\right| a-b \mid$ for any $a, b \in \mathbb{C}$, and the a priori bounds $\|\psi(t)\|_{\infty} \leqslant c$ and $\left\|\psi^{\varepsilon}(t)\right\|_{\infty} \leqslant c$, we get the inequality:

$$
\left|\psi^{\varepsilon}\left(t, y_{k}\right)-\psi\left(t, y_{k}\right)\right| \leqslant c \sum_{j=1}^{N} \int_{0}^{t} d s \frac{1}{\sqrt{t-s}}\left|\psi^{\varepsilon}\left(s, y_{j}\right)-\psi\left(s, y_{j}\right)\right|+c \varepsilon^{\delta} .
$$

By a standard argument in the theory of Abel integral operators, we conclude that

$$
\sup _{t \in[0, T]}\left|\psi^{\varepsilon}\left(t, y_{j}\right)-\psi\left(t, y_{j}\right)\right| \leqslant c \varepsilon^{\delta}
$$

for any $0<\delta<1 / 2$ and $T>0$. 
Step 3. Now we can proceed to the proof of the convergence in $L^{2}$-norm, precisely we shall show that

$$
\sup _{t \in[0, T]}\left\|\psi^{\varepsilon}(t)-\psi(t)\right\| \leqslant c \varepsilon^{\delta}
$$

for any $0<\delta<1 / 2$ and $T>0$.

We rewrite the nonlinear Eq. (9) explicitly, as

$$
\begin{aligned}
\psi^{\varepsilon}(t, x) & =\left(U(t) * \psi_{0}\right)(x) \\
& -i \sum_{j=1}^{N} \int_{0}^{t} d s \int_{\mathbb{R}} U\left(t-s, x-y_{j}-\varepsilon y\right) V_{j}(y)\left|\psi^{\varepsilon}\left(s, y_{j}+\varepsilon y\right)\right|^{2 \mu_{j}} \psi^{\varepsilon}\left(s, y_{j}+\varepsilon y\right) d y .
\end{aligned}
$$

As in Step 2, letting $\varepsilon \rightarrow 0$ at the r.h.s. we see that, if $\gamma_{j}=\int_{\mathbb{R}} V_{j} d x, \psi^{\varepsilon}(t, x)$ approximately satisfies the same equation as $\psi(t, x)$. To make precise this argument we subtract (17) from (5) and get the identity

$$
\begin{aligned}
& \psi^{\varepsilon}(t, x)-\psi(t, x)= \\
& -i \sum_{j=1}^{N} \gamma_{k} \int_{0}^{t} d s U\left(t-s, x-y_{j}\right)\left(\left|\psi^{\varepsilon}\left(s, y_{j}\right)\right|^{2 \mu_{j}} \psi^{\varepsilon}\left(s, y_{j}\right)-\left|\psi\left(s, y_{j}\right)\right|^{2 \mu_{j}} \psi\left(s, y_{j}\right)\right)+\mathcal{T}^{\varepsilon}(t, x) .
\end{aligned}
$$

where $\mathcal{T}^{\varepsilon}(t, x)$ is a remainder that satisfies the bound $\sup _{t \in[0, T]}\left\|\mathcal{T}^{\varepsilon}(t)\right\| \leqslant c \varepsilon^{\delta}$ for any $0<$ $\delta<1 / 2$ and $T>0$ (see [10] for the details). Taking the $L^{2}$-norm of $\psi^{\varepsilon}(t, x)-\psi(t, x)$ we obtain

$$
\begin{aligned}
& \sup _{t \in[0, T]}\left\|\psi^{\varepsilon}(t)-\psi(t)\right\| \\
\leqslant & \sum_{j=1}^{N}\left|\gamma_{j}\right| \sup _{t \in[0, T]}\left\|\int_{0}^{t} d s U\left(t-s, \cdot-y_{j}\right)\left(\left|\psi^{\varepsilon}\left(s, y_{j}\right)\right|^{2 \mu_{j}} \psi^{\varepsilon}\left(s, y_{j}\right)-\left|\psi\left(s, y_{j}\right)\right|^{2 \mu_{j}} \psi\left(s, y_{j}\right)\right)\right\|+c \varepsilon^{\delta} \\
\leqslant & \left.\sum_{j=1}^{N} \sup _{s \in[0, T]}|| \psi^{\varepsilon}\left(s, y_{j}\right)\right|^{2 \mu_{j}} \psi^{\varepsilon}\left(s, y_{j}\right)-\left|\psi\left(s, y_{j}\right)\right|^{2 \mu_{j}} \psi\left(s, y_{j}\right) \mid+c \varepsilon^{\delta} \\
\leqslant & c \sum_{j=1}^{N} \sup _{s \in[0, T]}\left|\psi^{\varepsilon}\left(s, y_{j}\right)-\psi\left(s, y_{j}\right)\right|+c \varepsilon^{\delta} \leqslant c \varepsilon^{\delta} .
\end{aligned}
$$

In the second inequality we used the bound

$$
\sup _{t \in[0, T]}\left\|\int_{0}^{t} U(t-s, \cdot) f(s) d s\right\| \leqslant c \sup _{s \in[0, T]}|f(s)| .
$$

In the third inequality we used $\left.|| a\right|^{2 \mu} a-|b|^{2 \mu} b\left|\leqslant\left(|a|^{2 \mu}+|b|^{2 \mu}\right)\right| a-b \mid$ and the a priori bounds $\|\psi(t)\|_{\infty} \leqslant c,\left\|\psi^{\varepsilon}(t)\right\|_{\infty} \leqslant c$. And finally we used the bound (15). This concludes the proof of $(16)$.

Step 4. We are left to prove the convergence in $H^{1}$. Since the bound $\left\|\psi^{\varepsilon^{\prime}}(t)\right\| \leqslant c$ holds true, there exists a subsequence, that we denote in the same way, such that $\psi^{\varepsilon}(t)$ converges weakly to $\phi(t)$ in $H^{1}(\mathbb{R})$. Recalling that $\psi^{\varepsilon}(t) \rightarrow \psi(t)$ in $L^{2}(\mathbb{R})$ we conclude that $\phi(t)=\psi(t)$ a.e.. Moreover, since:

$$
\left\|\psi^{\varepsilon}(t)-\psi(t)\right\|_{H^{1}}^{2}=\left\|\psi^{\varepsilon}(t)\right\|_{H^{1}}^{2}+\|\psi(t)\|_{H^{1}}^{2}-2 \operatorname{Re}\left(\psi^{\varepsilon}(t), \psi(t)\right)_{H^{1}},
$$

it is sufficient to prove that

$$
\lim _{\varepsilon \rightarrow 0}\left\|\psi^{\varepsilon}(t)\right\|_{H^{1}}^{2}=\|\psi(t)\|_{H^{1}}^{2}
$$


As in Step 3 we proved the convergence in $L^{2}$, we are left to show that

$$
\lim _{\varepsilon \rightarrow 0} \sup _{t \in[0, T]}\left|\left\|\psi^{\varepsilon \prime}(t)\right\|^{2}-\left\|\psi^{\prime}(t)\right\|^{2}\right|=0 .
$$

From the conservation of the energy we obtain

$$
\begin{aligned}
\left\|\psi^{\varepsilon \prime}(t)\right\|^{2} & =E^{\varepsilon}\left[\psi_{0}\right]-\sum_{j=1}^{N} \frac{1}{\mu_{j}+1} \int d x V_{j}^{\varepsilon}\left(x-y_{j}\right)\left|\psi^{\varepsilon}(t, x)\right|^{2 \mu_{j}+2} \\
\left\|\psi^{\prime}(t)\right\|^{2} & =E\left[\psi_{0}\right]-\sum_{j=1}^{N} \gamma_{j} \frac{1}{\mu_{j}+1}\left|\psi\left(t, y_{j}\right)\right|^{2 \mu_{j}+2} .
\end{aligned}
$$

Since $\lim _{\varepsilon \rightarrow 0} E^{\varepsilon}\left[\psi_{0}\right]=E\left[\psi_{0}\right]$, we have

$$
\begin{aligned}
& \lim _{\varepsilon \rightarrow 0} \sup _{t \in[0, T]}\left|\left\|\psi^{\varepsilon^{\prime}}(t)\right\|^{2}-\left\|\psi^{\prime}(t)\right\|^{2}\right| \\
= & \lim _{\varepsilon \rightarrow 0} \sup _{t \in[0, T]}\left|\sum_{j=1}^{N} \frac{1}{\mu_{j}+1}\left(\int_{\mathbb{R}} V_{j}(x)\left|\psi^{\varepsilon}\left(t, y_{j}+\varepsilon x\right)\right|^{2 \mu_{j}+2} d x-\gamma_{j}\left|\psi\left(t, y_{j}\right)\right|^{2 \mu_{j}+2}\right)\right| .
\end{aligned}
$$

By Step 2 we have $\left.\lim _{\varepsilon \rightarrow 0} \sup _{t \in[0, T]}|| \psi^{\varepsilon}\left(t, y_{j}\right)\right|^{2 \mu_{j}+2}-\left|\psi\left(t, y_{j}\right)\right|^{2 \mu_{j}+2} \mid=0$. Moreover, since $\gamma_{j}=\int_{\mathbb{R}} V_{j} d x$, it follows that

$$
\begin{aligned}
\left|\int_{\mathbb{R}} V_{j}(x)\right| \psi^{\varepsilon}\left(t, y_{j}\right. & +\varepsilon x)\left.\right|^{2 \mu_{j}+2} d x-\gamma_{j}\left|\psi^{\varepsilon}\left(t, y_{j}\right)\right|^{2 \mu_{j}+2} \mid \\
= & \left|\int_{\mathbb{R}} V_{j}(x)\left(\left|\psi^{\varepsilon}\left(t, y_{j}+\varepsilon x\right)\right|^{2 \mu_{j}+2}-\left|\psi^{\varepsilon}\left(t, y_{j}\right)\right|^{2 \mu_{j}+2}\right) d x\right| \\
& \leqslant c \sqrt{\varepsilon}\left\|\left|\psi^{\varepsilon}(t)\right|^{2 \mu_{k}+2}\right\|_{H^{1}} \int_{\mathbb{R}}\left|V_{j}(x)\right| \sqrt{|x|} d x \leqslant c \sqrt{\varepsilon} .
\end{aligned}
$$

The latter bound, together with the equality (19), give (18), and this concludes the proof of Th. 2.1.

\subsection{Remarks on the one dimensional problem}

We conclude the discussion on the one dimensional case with several remarks.

We note that the approximating problem (11) imitates the approximation result on the linear delta-interactions. Indeed it is well known, see [6], that the Hamiltonian $H^{\varepsilon}=-\Delta+\sum_{j} V_{j}^{\varepsilon}\left(\cdot-y_{j}\right)$, with $V_{j}^{\varepsilon}$ defined as in Eq. (10), converges in the norm resolvent sense to $H_{\alpha}$, defined by (1) - (2), with $\alpha_{j}=\int_{\mathbb{R}} V_{j} d x$. Recall that this also implies the convergence of associated unitary groups.

For the convergence of the linear problem one only needs to assume $V_{j} \in L^{1}(\mathbb{R})$. Also, the well-posedness result for Eq. (9) requires only $V_{j} \in L^{1}(\mathbb{R}) \cap L^{\infty}(\mathbb{R})$ (see [11]). This suggests that the assumption $V_{j} \in L^{1}(\mathbb{R},(1+|x|) d x) \cap L^{\infty}(\mathbb{R})$ in Th. (2.1) is not optimal.

Concerning the assumptions on $V_{j}$, we also note that the limit problem (5) is well posed for any $\mu_{j}>0$ if $\gamma_{j}>0$. Since $\gamma_{j}=\int_{\mathbb{R}} V_{j} d x$, one would expect the convergence result to hold true for any $\mu_{j}>0$ if $\int_{\mathbb{R}} V_{j} d x>0$. Nevertheless, the argument used to derive the uniform bound, $\left\|\psi^{\varepsilon \prime}(t)\right\| \leqslant c$ (step 1 in the proof of Th. 2.1), requires an upper bound on $\mu_{j}$ whenever $V_{j}$ is negative in some open interval. The same problem appears in the proof of the well-posedness of Eq. (9). This is a consequence of the fact that the argument neglects the positive part of $V_{j}$. 
In [16], the authors describe a different type of approximating problem which uses a non-local nonlinearity. They consider the equation

$$
i \frac{\partial}{\partial t} \psi^{\varepsilon}(t, x)=-\frac{\partial^{2}}{\partial x^{2}} \psi^{\varepsilon}(t, x)+\rho^{\varepsilon}(x) \alpha\left(\left|\left\langle\rho^{\varepsilon}, \psi^{\varepsilon}(t)\right\rangle\right|^{2}\right)\left\langle\rho^{\varepsilon}, \psi^{\varepsilon}(t)\right\rangle,
$$

where $\rho^{\varepsilon}$ is a function approximating the Dirac delta-distribution (i.e., $\rho^{\varepsilon}(x)=\rho(x / \varepsilon) / \varepsilon$ with $\rho \in C_{0}^{\infty}[-1,1], \rho \geqslant 0$, and $\left.\int_{\mathbb{R}} \rho d x=1\right)$ and $\alpha(z)$ is a nonlinear function.

Under suitable assumptions of the nonlinearity, they prove that, for any initial data in $H^{1}(\mathbb{R})$, the solution of Eq. $(20)$, converges to the solution of the equation $i \partial_{t} \psi=-\partial_{x x}^{2} \psi+$ $\alpha\left(|\psi(t, 0)|^{2}\right) \delta_{0} \psi$.

\section{Nonlinear delta-interactions in dimension three}

This section is devoted to the analysis of the approximation problem for the Schrödinger equation with nonlinear delta-interactions in dimension three. We will discuss a conjecture on an approximating problem with non-local nonlinearity. For the sake of simplicity we shall restrict the analysis to the case $N=1$. For a discussion on the generalization of the limit model to $N>1$ we refer to [1-3].

We shall start by recalling several results from [1] on the well-posedness of the limit model. Since we are setting $N=1$, we use the notation $\alpha_{1} \equiv \alpha$, and set $\mathbf{y}_{1} \equiv \mathbf{0}$, i.e., we put the center of interaction in the origin. We set

$$
G_{0}(\mathbf{x})=\frac{1}{4 \pi|\mathbf{x}|}
$$

In dimension three the operator formally written as $H_{\alpha}=-\Delta+\alpha \delta_{0}$ is defined by

$$
\begin{aligned}
D\left(H_{\alpha}\right)=\left\{\psi \in L^{2}\left(\mathbb{R}^{3}\right):\right. & \psi=\phi+q G_{0}, \phi \in H_{l o c}^{2}\left(\mathbb{R}^{3}\right), \\
& \nabla \phi \in L^{2}\left(\mathbb{R}^{3}\right), \Delta \phi \in L^{2}\left(\mathbb{R}^{3}\right), q \in \mathbb{C}, \\
& \left.\lim _{\mathbf{x} \rightarrow \mathbf{0}}\left(\psi(\mathbf{x})-q G_{0}(\mathbf{x})\right)=\alpha q\right\}
\end{aligned}
$$

and

$$
H_{\alpha} \psi=-\Delta \psi \quad \forall \mathbf{x} \neq 0 .
$$

The quantity $q$ is referred to as charge of the wave function $\psi$. We remark that by the definition of $D\left(H_{\alpha}\right)$, in general one has $\phi \notin H^{2}\left(\mathbb{R}^{3}\right)$. This is due to the decomposition $\psi=\phi+q G_{0}$ and to the fact that $G_{0} \notin L^{2}\left(\mathbb{R}^{3}\right)$. Whenever $q=0$, one has that $\psi=\phi$, and $\psi \in H^{2}(\mathbb{R})$ with $\psi(\mathbf{0})=0$.

The form domain of the operator $H_{\alpha}$ is

$$
V=\left\{\psi \in L^{2}\left(\mathbb{R}^{3}\right): \psi=\phi+q G_{0}, \phi \in H_{l o c}^{1}\left(\mathbb{R}^{3}\right), \nabla \phi \in L^{2}\left(\mathbb{R}^{3}\right), q \in \mathbb{C}\right\}
$$

As for the one dimensional case the form domain does not depend on $\alpha$. Yet, we note one main difference with the one dimensional case: $V$ does not coincide with $H^{1}(\mathbb{R})$, the form domain of the free Laplacian $\left(-\Delta, H^{2}\left(\mathbb{R}^{3}\right)\right)$. Then $H_{\alpha}$ is not a small perturbation, in the sense of quadratic forms, of $-\Delta$.

As for the one dimensional case, to give a precise definition of the nonlinear dynamics we are interested in, we start with a discussion on the non-autonomous Hamiltonian $H_{\alpha(t)}$, where $\alpha(t)$ is an assigned function. Such Hamiltonians have been studied, for example, in relation with the ionization problem (see, e.g., $[12,13]$ and references therein). 
Consider the Cauchy problem (3) in dimension three. From [20], one obtains its solution in terms of the time dependent charge $q(t)$. The charge satisfies the Volterra equation:

$$
\frac{q(t)}{4 \sqrt{\pi i}}+\int_{0}^{t} d s \frac{\alpha(s) q(s)}{\sqrt{t-s}}=\int_{0}^{t} d s \frac{\left(U(s) * \psi_{0}\right)(\mathbf{0})}{\sqrt{t-s}},
$$

where $U(t)$ is the unitary group generated by the free Laplacian in dimension three. Its explicit expression is given by

$$
U(t, \mathbf{x})=\frac{e^{i \frac{|\mathbf{x}|^{2}}{4 t}}}{(4 \pi i t)^{3 / 2}},
$$

while the solution $\psi(t)$ is completely defined by $q(t)$ through the equation

$$
\psi(t, \mathbf{x})=\left(U(t) * \psi_{0}\right)(\mathbf{x})+i \int_{0}^{t} d s U(t-s, \mathbf{x}) q(s) .
$$

The solutions defined by equations $(23)$ - (24) belong to $D\left(H_{\alpha(t)}\right)$ only under certain assumptions on the regularity of the function $\alpha(t)$ and on the initial state $\psi_{0}$, see, e.g., [21] and references therein for results in this direction.

To define a nonlinear delta-interaction in dimension three we let $\alpha(t) \rightarrow \alpha\left(|q(t)|^{2}\right)$ in equation (23). Following [1], we restrict ourselves to power type nonlinearities, and, as for the one dimensional case, we choose $\alpha(z)=\gamma z^{\mu}$, with $\gamma \in \mathbb{R}$ and $\mu>0$.

With this choice the linear Volterra equation (23) is replaced by the nonlinear one

$$
\frac{q(t)}{4 \sqrt{\pi i}}+\gamma \int_{0}^{t} d s \frac{|q(s)|^{2 \mu} q(s)}{\sqrt{t-s}}=\int_{0}^{t} d s \frac{\left(U(s) * \psi_{0}\right)(\mathbf{0})}{\sqrt{t-s}} .
$$

Equation (24) - (25), define our model for a nonlinear delta-interaction in dimension three. We recall that, global well-posedness for the problem (24) - (25) in $\psi_{0} \in V$ and under the condition that $\mu<1$ if $\gamma<0$ was proved in [1]. In the same paper, the authors also prove that the nonlinear flow admits two conserved quantities: the $L^{2}$-norm (mass) and the energy

$$
E[\psi]=\|\nabla \phi\|^{2}+\frac{\gamma|q|^{2 \mu+2}}{\mu+1},
$$

where $\psi=\phi+q G_{0} \in V$. So that, for any $\psi_{0} \in V$, if $\psi(t)$ is the solution of the problem (24) - (25), one has $\|\psi(t)\|^{2}=\left\|\psi_{0}\right\|^{2}$ and $E[\psi(t)]=E\left[\psi_{0}\right]$.

We remark that by the definition of the form domain of $H_{\alpha}, \psi \in V$ does not imply $\psi \in H^{1}\left(R^{3}\right)$, and the well-posedness in $V$ must be understood in the sense that $\|\nabla \phi(t)\|$ and $|q(t)|$ stay bounded.

We also remark that in [1], the authors consider the more general case of finitely many nonlinear delta-interactions, $N \geqslant 1$. The conditions on the initial state which guarantee $\psi(t) \in D\left(H_{\alpha\left(|q(t)|^{2}\right)}\right)$ are also discussed in [1]. We refer to [2] for the analysis of the blowup problem and to [3] for the study of the stability/instability properties of the stationary solutions of $(24)-(25)$.

\subsection{Point-like limit of scaled non-local nonlinearities in dimension three}

In this section we discuss a conjecture about the approximation problem for (24) - (25)through scaled non-local nonlinear flows. We remark that in dimension three the problem of finding an approximation of the nonlinear flow (24) - (25) is subtler than the corresponding problem in dimension one. This is not surprising, as also the problem of finding a regular approximation of the (linear) operator $H_{\alpha}$ in dimension three requires non-trivial renormalization procedures (see, [6]). 
Let $\rho$ be such that $\rho \in C_{0}^{\infty}\left(\mathbb{R}^{3}\right), \rho \geqslant 0$, and $\int_{\mathbb{R}^{3}} \rho \mathrm{d} \mathbf{x}=1$, then set $\rho^{\varepsilon}(\mathbf{x})=\rho(\mathbf{x} / \varepsilon) / \varepsilon^{3}$, so that $\rho^{\varepsilon}$ converges to the Dirac delta-distribution in dimension three.

We denote by $\tilde{\rho}(\mathbf{k})$ and $\tilde{\rho}^{\varepsilon}(\mathbf{k})$ the Fourier transform of $\rho$ and $\rho^{\varepsilon}$ respectively, by scaling one has that $\tilde{\rho}^{\varepsilon}(\mathbf{k})=\tilde{\rho}(\varepsilon \mathbf{k})$. Moreover, from $\int_{\mathbb{R}^{3}} \rho \mathrm{d} \mathbf{x}=1$, it follows that $\tilde{\rho}(\mathbf{0})=1$.

We set $M=\frac{1}{(2 \pi)^{3}} \int_{\mathbb{R}^{3}} \mathrm{~d} \mathbf{k}(\tilde{\rho}(\mathbf{k}))^{2} /|\mathbf{k}|^{2}$ and consider the nonlinear flow (in weak form)

$$
\begin{aligned}
\psi^{\varepsilon}(t, \mathbf{x})=( & \left.U(t) * \psi_{0}^{\varepsilon}\right)(\mathbf{x}) \\
& -i \frac{\varepsilon}{M} \int_{0}^{t} d s\left(U(t-s) * \rho^{\varepsilon}\right)(\mathbf{x})\left(-1+\gamma \frac{\varepsilon^{2 \mu+1}\left|\left\langle\rho^{\varepsilon}, \psi^{\varepsilon}(s)\right\rangle\right|^{2 \mu}}{M^{2 \mu+1}}\right)\left\langle\rho^{\varepsilon}, \psi^{\varepsilon}(s)\right\rangle .
\end{aligned}
$$

Note that we let the initial datum $\psi_{0}^{\varepsilon}$ to depend on $\varepsilon$ as well. This is due to the fact that we want to compare the solutions of Eq. (26) with the solution of (24) - (25). To do that, one should consider the two nonlinear flows with the same initial datum. In dimension three this is, in general, not possible, because problem (24) - (25) is naturally defined for initial data in $V$, while problem (26) is naturally defined for initial data in $H^{1}\left(\mathbb{R}^{3}\right)$. For this reason we let the initial datum in $(26)$ to depend on $\varepsilon$, and assume that $\psi_{0}^{\varepsilon}$ converges to $\psi_{0}$ in a suitable norm as $\varepsilon \rightarrow 0$.

To begin, it is also convenient to set $\gamma>0$ (defocusing nonlinearity), this choice simplifies some issues with the well-posedness, giving at once some useful bounds on relevant quantities, and avoids further constraints on the power of the nonlinearity $\mu$.

We remark that problem (26), is a weak formulation of

$$
\left\{\begin{array}{l}
i \frac{\partial}{\partial t} \psi^{\varepsilon}(t, \mathbf{x})=-\Delta \psi^{\varepsilon}(t, \mathbf{x})+\frac{\varepsilon}{M}\left(-1+\gamma \frac{\varepsilon^{2 \mu+1}\left|\left\langle\rho^{\varepsilon}, \psi^{\varepsilon}(t)\right\rangle\right|^{2 \mu}}{M^{2 \mu+1}}\right)\left\langle\rho^{\varepsilon}, \psi^{\varepsilon}(t)\right\rangle \rho^{\varepsilon}(\mathbf{x}) \\
\psi^{\varepsilon}(0, \mathbf{x})=\psi_{0}^{\varepsilon}(\mathbf{x})
\end{array}\right.
$$

We also note that, also in this case, the nonlinear approximation problem is inspired by the linear one. Indeed, it is easy prove that the (non-local) operator $H^{\varepsilon}=-\Delta+$ $\frac{\varepsilon}{M}\left(-1+\alpha \frac{\varepsilon}{M}\right)\left\langle\rho^{\varepsilon}, \cdot\right\rangle \rho^{\varepsilon}$ converges in norm resolvent sense to the operator $H_{\alpha}$ defined by $(21)$ $-(22)$.

We conjecture that, for any $\psi_{0} \in V$ and $\psi_{0}^{\varepsilon} \in H^{1}\left(\mathbb{R}^{3}\right)$ that converges to $\psi_{0}$ in a suitable sense, the solution of Eq. (26) converges to the solution of (24) - (25).

We do not have yet a rigorous proof of this statement, but we are able to give an heuristic argument to support the conjecture.

The basic idea is that the quantity $q^{\varepsilon}(t)=\frac{\varepsilon}{M}\left\langle\rho^{\varepsilon}, \psi^{\varepsilon}(t)\right\rangle$, should converge to the charge $q(t)$. Eq. (26), written in terms of $q^{\varepsilon}(t)$, gives

$$
\psi^{\varepsilon}(t, \mathbf{x})=\left(U(t) * \psi_{0}^{\varepsilon}\right)(\mathbf{x})-i \int_{0}^{t} d s\left(U(t-s) * \rho^{\varepsilon}\right)(\mathbf{x})\left(-1+\gamma \frac{\varepsilon}{M}\left|q^{\varepsilon}(s)\right|^{2 \mu}\right) q^{\varepsilon}(s) .
$$

We recall that $\rho^{\varepsilon} \rightarrow \delta_{0}$, then $\left(U(t-s) * \rho^{\varepsilon}\right)(\mathbf{x}) \rightarrow U(t-s, \mathbf{x})$. Assuming, moreover, that $U(t) * \psi_{0}^{\varepsilon} \rightarrow U(t) * \psi_{0}$, one expects that

$$
\psi^{\varepsilon}(t, \mathbf{x}) \simeq\left(U(t) * \psi_{0}\right)(\mathbf{x})+i \int_{0}^{t} d s U(t-s, \mathbf{x}) q^{\varepsilon}(s) .
$$

By comparison with Eq. (24), this suggests that, if $q^{\varepsilon}(t)$ converges to $q(t)$, then also $\psi^{\varepsilon}(t)$ converges to $\psi(t)$. 
To support the idea that $q^{\varepsilon}(t)$ should converge to $q(t)$ we proceed as follows. We take the scalar product of Eq. (27) with $\rho^{\varepsilon}$, using the definition of $q^{\varepsilon}(t)$ we get the identity

$$
\frac{M}{\varepsilon} q^{\varepsilon}(t)=\left\langle\rho^{\varepsilon}, U(t) * \psi_{0}^{\varepsilon}\right\rangle-i \int_{0}^{t} d s\left\langle\rho^{\varepsilon}, U(t-s) * \rho^{\varepsilon}\right\rangle\left(-1+\gamma \frac{\varepsilon}{M}\left|q^{\varepsilon}(s)\right|^{2 \mu}\right) q^{\varepsilon}(s) .
$$

Let $K^{\varepsilon}(t)=\frac{1}{(2 \pi)^{3}} \int_{\mathbb{R}^{3}} \mathrm{~d} \mathbf{k}\left(\tilde{\rho}^{\varepsilon}(\mathbf{k})\right)^{2} e^{-i|\mathbf{k}|^{2} t} /|\mathbf{k}|^{2}$. By the definition of $U(t)$ one has that

$$
\left\langle\rho^{\varepsilon}, U(t) * \rho^{\varepsilon}\right\rangle=\frac{1}{(2 \pi)^{3}} \int_{\mathbb{R}^{3}} \mathrm{~d} \mathbf{k}\left(\tilde{\rho}^{\varepsilon}(\mathbf{k})\right)^{2} e^{-i|\mathbf{k}|^{2} t}=\frac{i}{(2 \pi)^{3}} \frac{d}{d t} \int_{\mathbb{R}^{3}} \mathrm{~d} \mathbf{k} \frac{\left(\tilde{\rho}^{\varepsilon}(\mathbf{k})\right)^{2} e^{-i|\mathbf{k}|^{2} t}}{|\mathbf{k}|^{2}}=i \frac{d}{d t} K^{\varepsilon}(t) .
$$

Using the latter identity, written as $\left\langle\rho^{\varepsilon}, U(t-s) * \rho^{\varepsilon}\right\rangle=-i \frac{d}{d s} K^{\varepsilon}(t-s)$, in (28), and by integration by parts in $s$, we get

$$
\begin{aligned}
\frac{M}{\varepsilon} q^{\varepsilon}(t)=\left\langle\rho^{\varepsilon}, U(t)\right. & \left.* \psi_{0}^{\varepsilon}\right\rangle \\
& +\int_{0}^{t} d s K^{\varepsilon}(t-s) \frac{d}{d s}\left[\left(-1+\gamma \frac{\varepsilon}{M}\left|q^{\varepsilon}(s)\right|^{2 \mu}\right) q^{\varepsilon}(s)\right] \\
& -K^{\varepsilon}(0)\left(-1+\gamma \frac{\varepsilon}{M}\left|q^{\varepsilon}(t)\right|^{2 \mu}\right) q^{\varepsilon}(t)+K^{\varepsilon}(t)\left(-1+\gamma \frac{\varepsilon}{M}\left|q^{\varepsilon}(0)\right|^{2 \mu}\right) q^{\varepsilon}(0) .
\end{aligned}
$$

Using the identity $K^{\varepsilon}(0)=M / \varepsilon$ we see that $\frac{M}{\varepsilon} q^{\varepsilon}(t)$ cancels, so that, rearranging the terms, we are left with

$$
\begin{aligned}
\gamma\left|q^{\varepsilon}(t)\right|^{2 \mu} q^{\varepsilon}(t)=\left\langle\rho^{\varepsilon}, U(t) * \psi_{0}^{\varepsilon}\right\rangle+\int_{0}^{t} d s K^{\varepsilon}(t-s) \frac{d}{d s} & {\left[\left(-1+\gamma \frac{\varepsilon}{M}\left|q^{\varepsilon}(s)\right|^{2 \mu}\right) q^{\varepsilon}(s)\right] } \\
& +K^{\varepsilon}(t)\left(-1+\gamma \frac{\varepsilon}{M}\left|q^{\varepsilon}(0)\right|^{2 \mu}\right) q^{\varepsilon}(0) .
\end{aligned}
$$

To recover an equation of the form (25), we multiply for $\frac{1}{\sqrt{\tau-t}}$ and integrate in $t$ for $t \in[0, \tau]$. We obtain

$$
\begin{aligned}
\gamma \int_{0}^{\tau} d t \frac{\left|q^{\varepsilon}(t)\right|^{2 \mu} q^{\varepsilon}(t)}{\sqrt{\tau-t}} & =\int_{0}^{\tau} d t \frac{\left\langle\rho^{\varepsilon}, U(t) * \psi_{0}^{\varepsilon}\right\rangle}{\sqrt{\tau-t}} \\
+\int_{0}^{\tau} d t & \frac{1}{\sqrt{\tau-t}} \int_{0}^{t} d s K^{\varepsilon}(t-s) \frac{d}{d s}\left[\left(-1+\gamma \frac{\varepsilon}{M}\left|q^{\varepsilon}(s)\right|^{2 \mu}\right) q^{\varepsilon}(s)\right] \\
& +\left(-1+\gamma \frac{\varepsilon}{M}\left|q^{\varepsilon}(0)\right|^{2 \mu}\right) q^{\varepsilon}(0) \int_{0}^{\tau} d t \frac{K^{\varepsilon}(t)}{\sqrt{\tau-t}} .
\end{aligned}
$$

The heuristic argument showing that $q^{\varepsilon}(t)$ should converge to $q(t)$ is based on the fact that

$$
K^{\varepsilon}(t) \rightarrow \frac{1}{(2 \pi)^{3}} \int_{\mathbb{R}^{3}} \mathrm{~d} \mathbf{k} \frac{e^{-i|\mathbf{k}|^{2} t}}{|\mathbf{k}|^{2}}=\frac{1}{4 \pi \sqrt{\pi i} \sqrt{t}},
$$

as $\varepsilon \rightarrow 0$. This suggests that Eq. (29) should be approximated by

$$
\begin{aligned}
& \gamma \int_{0}^{\tau} d t \frac{\left|q^{\varepsilon}(t)\right|^{2 \mu} q^{\varepsilon}(t)}{\sqrt{\tau-t}} \simeq \int_{0}^{\tau} d t \frac{\left\langle\rho^{\varepsilon}, U(t) * \psi_{0}^{\varepsilon}\right\rangle}{\sqrt{\tau-t}} \\
&+\frac{1}{4 \pi \sqrt{\pi i}} \int_{0}^{\tau} d t \frac{1}{\sqrt{\tau-t}} \int_{0}^{t} d s \frac{1}{\sqrt{t-s}} \frac{d}{d s}\left[\left(-1+\gamma \frac{\varepsilon}{M}\left|q^{\varepsilon}(s)\right|^{2 \mu}\right) q^{\varepsilon}(s)\right] \\
&+\frac{1}{4 \pi \sqrt{\pi i}}\left(-1+\gamma \frac{\varepsilon}{M}\left|q^{\varepsilon}(0)\right|^{2 \mu}\right) q^{\varepsilon}(0) \int_{0}^{\tau} d t \frac{1}{\sqrt{\tau-t}} \frac{1}{\sqrt{t}} .
\end{aligned}
$$


In the second line of the equation above we exchange the integrals in $t$ and $s$ and use the fact that $\int_{0}^{\tau} d t \frac{1}{\sqrt{\tau-t}} \frac{1}{\sqrt{t-s}} \mathbf{1}_{\{t>s\}}=\pi$, while in the third line we use $\int_{0}^{\tau} d t \frac{1}{\sqrt{\tau-t}} \frac{1}{\sqrt{t}}=\pi$, to get

$$
\begin{aligned}
\gamma \int_{0}^{\tau} d t \frac{\left|q^{\varepsilon}(t)\right|^{2 \mu} q^{\varepsilon}(t)}{\sqrt{\tau-t}} & \simeq \int_{0}^{\tau} d t \frac{1}{\sqrt{\tau-t}}\left\langle\rho^{\varepsilon}, U(t) * \psi_{0}^{\varepsilon}\right\rangle \\
& +\frac{1}{4 \sqrt{\pi i}} \int_{0}^{\tau} d s \frac{d}{d s}\left[\left(-1+\gamma \frac{\varepsilon}{M}\left|q^{\varepsilon}(s)\right|^{2 \mu}\right) q^{\varepsilon}(s)\right] \\
& \quad+\frac{1}{4 \sqrt{\pi i}}\left(-1+\gamma \frac{\varepsilon}{M}\left|q^{\varepsilon}(0)\right|^{2 \mu}\right) q^{\varepsilon}(0)
\end{aligned}
$$

Performing the integral in $s$, and neglecting further terms of order $\varepsilon$, we finally end up with

$$
\gamma \int_{0}^{\tau} d t \frac{\left|q^{\varepsilon}(t)\right|^{2 \mu} q^{\varepsilon}(t)}{\sqrt{\tau-t}} \simeq \int_{0}^{\tau} d t \frac{\left\langle\rho^{\varepsilon}, U(t) * \psi_{0}^{\varepsilon}\right\rangle}{\sqrt{\tau-t}}-\frac{q^{\varepsilon}(\tau)}{4 \sqrt{\pi i}}
$$

Comparing the latter formula with Eq. (25), one sees that $q^{\varepsilon}(t)$ satisfies approximatively the same equation as $q(t)$.

To make rigorous the heuristic argument described above is the goal of a forthcoming work. One would expect that the right approach is to add and subtract to $K^{\varepsilon}$, in Eq. (29), its limit, given in formula (30), then prove that the remainder (the term containing the difference between $K^{\varepsilon}$ and its limit) is small. One main issue with this approach is the presence of the derivative of $q^{\varepsilon}(s)$ in Eq. (29), which implies that the remainder depends on $\dot{q}^{\varepsilon}$. The dependence of the reminder on $\dot{q}^{\varepsilon}$ is unexpected, because the limit equation does not involve the derivative of $q$, and difficult to treat, because it requires a bound on $\dot{q}^{\varepsilon}$. An approach that avoids taking the derivative of $q^{\varepsilon}$ would be preferable.

\section{Conclusions}

We addressed the problem of finding regular approximations of nonlinear deltainteractions.

We showed that, in dimension one, it is possible to find both local (see [10]) and non-local (see [16]) nonlinearities that converge to the limit problem. The convergence can be rigorously proved for initial data in $H^{1}(\mathbb{R})$.

For what concerns the three dimensional problem, we suggested an approximation through non-local nonlinearities. The convergence to the limit problem seems to be plausible but it is not yet proved rigorously.

Several problems remain completely open and would deserve some attention. We mention two of them which are strictly related. They concern the approximation of deltainteractions in dimension one and three, through local nonlinearities in the presence of a linear term with a zero energy resonance.

The approximation of $H_{\alpha}$ in dimension three through Hamiltonians with scaled potentials is not as intuitive as in dimension one. We already noted that in dimension one the operator $-\Delta+V(\cdot / \varepsilon) / \varepsilon$ converges to $H_{\alpha}$, with $\alpha=\int_{\mathbb{R}} V d x$. One might then think that also in dimension three, adding a delta-convergent function to the Laplacian would give an operator that converges to $H_{\alpha}$. This does not happen to be the case.

To define a sequence of Schrödinger operators that converge to $H_{\alpha}$ one has to choose a potential $V$ such that the operator $H=-\Delta+V$ has a zero energy resonance. That means that there exists a function $\phi$ such that $\phi \in L_{l o c}^{2}\left(\mathbb{R}^{3}\right), \nabla \phi \in L^{2}(\mathbb{R})$ and $H \phi=0$ in 
the distributional sense. Assuming additionally, that $V$ decays fast enough, one has that (see [6])

$$
H^{\varepsilon}=-\Delta+\frac{1+\lambda \varepsilon}{\varepsilon^{2}} V\left(\frac{\cdot}{\varepsilon}\right)
$$

where $\lambda \in \mathbb{R}$ converges (in norm resolvent sense) to $H_{\alpha}$, with coupling constant $\alpha=-m \lambda$, where $m$ is a positive constant that depends on $V$.

One may wonder whether letting $\lambda \rightarrow \lambda(\psi)$ would allow to obtain a local approximation of the nonlinear delta-interaction in dimension three.

A similar problem can be formulated in dimension one. Assume that $V$ decays fast enough and that it is such that $H=-\Delta+V$ has a zero energy resonance, i.e., there exists $\phi \in L^{\infty}(\mathbb{R}), \phi \notin L^{2}(\mathbb{R})$, such that $-\phi^{\prime \prime}+V \phi=0$. Assume also that the resonance $\phi$ is an even function. Then the operator $H^{\varepsilon}$ in (31), in dimension one, converges in norm resolvent sense to $H_{\alpha}$, defined in (1) - (2), with $\alpha=-\widetilde{m} \lambda$, where $\widetilde{m}$ is a positive constant which depends on $V$ (see [9]). In this sense the problems of approximating $H_{\alpha}$ in dimension one and three are similar. We remark that, in dimension one, also the convergence of the operator $-\Delta+V(\cdot / \varepsilon) / \varepsilon$ relies on the presence of a zero energy resonance of the operator $\left(-\Delta, H^{2}(\mathbb{R})\right)$. In this case the resonance is just the constant function.

Also in the one dimensional case, one might expect that letting $\lambda \rightarrow \lambda(\psi)$ in (31) will allow one to construct an approximation of a nonlinear delta-interaction.

\section{Acknowledgements}

The author is grateful to his friends and collaborators Domenico Finco, Diego Noja, and Alessandro Teta, all the results and conjectures presented in this report were obtained and formulated in collaboration with them. The support of the FIR 2013 project "Condensed Matter in Mathematical Physics" (code RBFR13WAET) is also acknowledged.

\section{References}

[1] Adami, R., Dell'Antonio, G., Figari, R., and Teta, A., The Cauchy problem for the Schrödinger equation in dimension three with concentrated nonlinearity, Ann. Inst. H. Poincaré Anal. Non Linéaire 20 (2003), no. 3, 477-500.

[2] Adami, R., Dell'Antonio, G., Figari, R., and Teta, A., Blow-up solutions for the Schrödinger equation in dimension three with a concentrated nonlinearity, Ann. Inst. H. Poincaré Anal. Non Linéaire 21 (2004), no. 1, 121-137.

[3] Adami, R., Noja, D., and Ortoleva, C., Orbital and asymptotic stability for standing waves of a nonlinear Schrödinger equation with concentrated nonlinearity in dimension three, J. Math. Phys. 54 (2013), no. 1, 013501.

[4] Adami, R. and Teta, A., A Simple Model of Concentrated Nonlinearity, Mathematical Results in Quantum Mechanics (Dittrich, J., Exner, P., and Tater, M., eds.), Operator Theory Advances and Applications, Birkhäuser Basel, January 1999, pp. 183-189.

[5] Adami, R. and Teta, A., A class of nonlinear Schrödinger equations with concentrated nonlinearity, J. Funct. Anal. 180 (2001), no. 1, 148-175.

[6] Albeverio, S., Gesztesy, F., Hoegh-Krohn, R., and Holden, H., Solvable Models in Quantum Mechanics, AMS, 2005.

[7] Berezin, F. A. and Faddeev, L. D., A Remark on Schrödinger's equation with a point interaction, Soviet Math. Dokl. 2 (1961), 372-375.

[8] Bulashenko, O., Kochelap, V., and Bonilla, L., Coherent patterns and self-induced diffraction of electrons on a thin nonlinear layer, Phys. Rev. B 54 (1996), no. 3, 1537-1540.

[9] Cacciapuoti, C. and Exner, P., Nontrivial edge coupling from a Dirichlet network squeezing: the case of a bent waveguide, J. Phys. A: Math. Theor. 40 (2007), no. 26, F511-F523. 
[10] Cacciapuoti, C., Finco, D., Noja, D., and Teta, A., The NLS Equation in Dimension One with Spatially Concentrated Nonlinearities: the Pointlike Limit, Lett. Math. Phys. 104 (2014), no. 12, 1557-1570.

[11] Cazenave, T., Semilinear Schrödinger Equations, Courant Lect. Notes Math. ed., vol. 10, AMS, 2003.

[12] Correggi, M. and Dell'Antonio, G., Decay of a bound state under a time-periodic perturbation: a toy case, J. Phys. A 38 (2005), no. 22, 4769-4781.

[13] Correggi, M., Dell'Antonio, G., Figari, R., and Mantile, A., Ionization for Three Dimensional TimeDependent Point Interactions, Comm. Math. Phys. 257 (2005), no. 1, 169-192.

[14] Dror, N. and Malomed, B. A., Solitons supported by localized nonlinearities in periodic media, Phys. Rev. A 83 (2011), no. 3, 033828.

[15] Hmidi, T., Mantile, A., and Nier, F., Time-dependent Delta-interactions for $1 D$ Schrödinger Hamiltonians, Math. Phys. Anal. Geom. 13 (2009), no. 1, 83-103.

[16] Komech, A. I. and Komech, A. A., Global well-posedness for the Schrödinger equation coupled to a nonlinear oscillator, Russ. J. Math. Phys. 14 (2007), no. 2, 164-173.

[17] Kronig, R., de L. and Penney, W. G., Quantum mechanics of electrons in crystal lattices, Proc. R. Soc. Lond. Ser. A 130 (1931), no. 814, 499-513.

[18] Malomed, B. A. and Azbel, M. Y., Modulational instability of a wave scattered by a nonlinear center, Phys. Rev. B 47 (1993), no. 16, 10402-10406.

[19] Neidhardt, H., Zagrebnov, V. A., et al., Linear non-autonomous Cauchy problems and evolution semigroups, Adv. Differential Equations 14 (2009), no. 3/4, 289-340.

[20] Sayapova, M. R. and Yafaev, D. R., The evolution operator for time-dependent potentials of zero radius, Boundary value problems of mathematical physics, vol. 159, Part 12, Work collection, Trudy Mat. Inst. Steklov., 1983, pp. 167-174.

[21] Yafaev, D. R., Scattering theory for time-dependent zero-range potentials, Annales de l'IHP Physique théorique 40 (1984), no. 4, 343-359. 